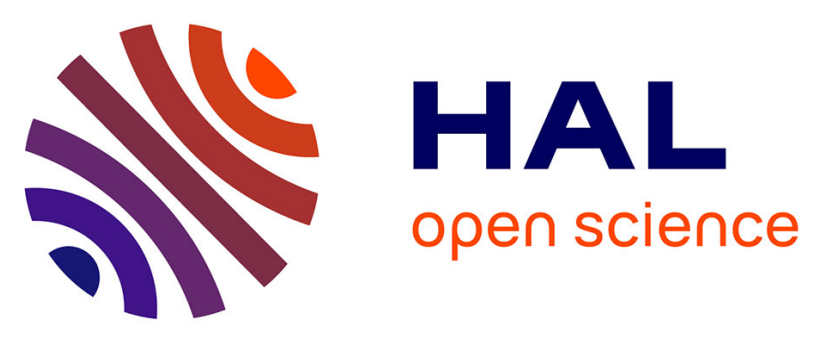

\title{
La résistance du piment (Capsicum annuum L.) à Phytophthora capsici Leon. XI. Réponse de 5 lignées de piment à une " fraction élicitrice"; influence de la dose d'éliciteur et efficacité de la protection induite vis-à-vis de plusieurs souches du parasite
}

Paul-Michel Molot, E. Pochard, Pierre Mas

\section{To cite this version:}

Paul-Michel Molot, E. Pochard, Pierre Mas. La résistance du piment (Capsicum annuum L.) à Phytophthora capsici Leon. XI. Réponse de 5 lignées de piment à une " fraction élicitrice "; influence de la dose d'éliciteur et efficacité de la protection induite vis-à-vis de plusieurs souches du parasite. Agronomie, 1983, 3 (4), pp.327-332. 10.1051/agro:19830405 . hal-02726840

\author{
HAL Id: hal-02726840 \\ https://hal.inrae.fr/hal-02726840
}

Submitted on 2 Jun 2020

HAL is a multi-disciplinary open access archive for the deposit and dissemination of scientific research documents, whether they are published or not. The documents may come from teaching and research institutions in France or abroad, or from public or private research centers.
L'archive ouverte pluridisciplinaire HAL, est destinée au dépôt et à la diffusion de documents scientifiques de niveau recherche, publiés ou non, émanant des établissements d'enseignement et de recherche français ou étrangers, des laboratoires publics ou privés. 


\title{
La résistance du piment (Capsicum annuum L.) à Phytophthora capsici Leon. XI. Réponse de 5 lignées de piment à une "fraction élicitrice "; influence de la dose d'éliciteur et efficacité de la protection induite vis-à-vis de plusieurs souches du parasite.
}

\author{
Paul-Michel MOLOT, Edmond POCHARD (*) \& Pierre MAS, \\ I.N.R.A., Station de Pathologie végétale, \\ (*) Station d'Amélioration des Plantes, Centre de Recherches d'Avignon, F 84140 Montfavet.
}

Mots-clés additionnels : Résistance induite, Eliciteur, Dose d'inoculum, Souches de champignon, Variétés de piment.

Additional key-words : Induced resistance, Elicitor, Inoculum quantity, Isolates, Pepper lines.

\section{INTRODUCTION}

A partir des hyphes ou du filtrat de culture de Phytophthora capsici Leon, il est possible, soit par précipitation à l'éthanol (Molot et al., 1980 b), soit par dépôt d'un culot de mycélium broyé à la surface d'un gradient de densité discontinu de saccharose (COULOMB et al., 1980), d'obtenir des fractions capables d'induire une protection efficace des cotylédons de piment «Yolo Wonder » en survie vis-à-vis des zoospores de $P$. capsici. 
Parmi ces différentes fractions, nous avons choisi la fraction G4 dont nous avons étudié par ailleurs (MOLOT et al., 1980 b) quelques propriétés biologiques.

Disposant d'un test biologique simple et rapide, réalisé sur organes foliaires en survie sur l'eau, nous nous proposons de mieux définir les conditions d'action de cette fraction en fonction des paramètres suivants : quantité de $\mathrm{G} 4$, choix de la souche contaminatrice, dose d'inoculum utilisée, choix de la variété de piment.

\section{MATÉRIEL ET MÉTHODES}

\section{A. Matériel végétal}

Cinq lignées de piment ont été utilisées dont une, «Yolo Wonder ", sensible à $P$. capsici et 4 autres partiellement résistantes : «PM 217 » et « Phyo 636 » (issues du même géniteur 493-1) ainsi que « L 29 » et « CM 334 » d'origine mexicaine (GUERERRO-MORENO \& LABORDE, 1980).

L'expérimentation est conduite sur organes foliaires prélevés soit au stade plantule (piment avec 2 cotylédons et 2 jeunes feuilles), soit au stade adulte, la plante présentant des ébauches florales. On recueille alors les 2 feuilles complètement développées, situées sous le « bouquet » terminal.

\section{B. Obtention de la fraction G4}

Elle est préparée à partir de la souche monozoospore 15-12-A de $P$. capsici. Le champignon est cultivé pendant 6 semaines sur bouillon de pomme de terre non agité. Le filtrat de culture recueilli est fractionné selon le type $\mathrm{G}$ : après concentration et précipitation à l'éthanol à $95^{\circ}$ (4 vol. d'éthanol pour 1 vol. de filtrat concentré 10 fois), on obtient une partie soluble dans l'alcool que l'on dialyse contre l'eau ; G4 correspond au rétentat insoluble dans l'eau et retenu dans le « boudin » (MoLOT et al., 1980 b).

\section{Mode d'application de la fraction G4}

Qu'il s'agisse de cotylédons ou de véritables feuilles, les organes foliaires sont, aussitôt après leur prélèvement, mis en survie sur l'eau, la face inférieure étant dirigée vers le haut. La fraction G4 est déposée au centre du limbe sous forme d'une goutte unique de $25 \mu \mathrm{l}$, représentant respectivement $3,6,12$ et $24 \mu \mathrm{g} /$ organe foliaire. Les témoins reçoivent $25 \mu$ l d'eau.

Chaque traitement répété 3 fois se fait sur des lots de 16 cotylédons (ou jeunes feuilles) et 12 feuilles adultes.

\section{Contamination artificielle}

Après $24 \mathrm{~h}$ (temps d'absorption de la goutte et de mise en place de l'induction), on pratique une contamination artificielle par $P$. capsici selon 2 méthodes :

- les organes foliaires issus de plantules reçoivent, au site de dépôt de la fraction $\mathrm{G} 4,10 \mu \mathrm{l}$ d'une suspension de zoospores de la souche 15 de $P$. capsici renfermant 80000 , 500000 ou 1000000 de zoospores / $\mathrm{ml}$. La contamination par zoospores se fait sans blessure.

- les feuilles issues de plantes adultes sont contaminées par un fragment calibré ( $\phi 4 \mathrm{~mm}$ ) de culture mycélienne sur milieu V8 (milieu Messiaen, composé de divers jus de légumes.). La contamination s'effectue toujours sur une bles- sure pratiquée avec une aiguille lancéolée au site de dépôt de G4. Huit souches de $P$. capsici, d'agressivité variable, ont été choisies : 13, 15, 75, 91, 94, 101, 107 et 197.

\section{E. Notations}

Les symptômes commencent à apparaître environ 3 jours après la contamination et font l'objet de notations quotidiennes pendant 1 semaine. Dans un but de simplification, nous ne mentionnons ici que les résultats de la dernière notation.

Selon une méthode déjà décrite (MoLot et al., 1980 b), l'indice de sensibilité des cotylédons ou des jeunes feuilles est exprimé en p. 100,100 p. 100 correspondant à la destruction totale du matériel végétal.

Sur les feuilles adultes, on mesure le diamètre de la zone attaquée par le champignon (MOLOT et al., 1980 a) ; les résultats sont traduits en p. 100 du témoin (sans fraction G4).

L'interprétation statistique des résultats est réalisée selon le test de Duncan.

\section{RÉSULTATS}

\section{A. Influence de la quantité de G4 et de la dose d'inocu- lum sur l'expression de la résistance induite (tabl. 1)}

Cette expérimentation est conduite sur cotylédons et jeunes feuilles de « Yolo Wonder » et « Phyo 636 » contaminés par zoospores.

Chez le témoin, les feuilles sont, dans l'ensemble, aussi sensibles sinon légèrement plus que les cotylédons. Malgré cela, après application de $\mathrm{G} 4$, elles se révèlent moins attaquées que les cotylédons. La sensibilité des organes foliaires apparaît sous la dépendance de 2 facteurs :

- d'une part la quantité de G4. L'effet de protection optimum s'observe à la concentration de $12 \mu \mathrm{g}$; à dose plus forte $24 \mu \mathrm{g}$ et surtout plus faible $3 \mu \mathrm{g}$, le matériel végétal devient plus sensible.

- d'autre part le nombre de zoospores. Plus celui-ci augmente, plus les dommages augmentent. Le niveau de résistance acquise peut être partiellement surmonté par des doses massives d'inoculum.

Ces résultats concernent les 2 variétés utilisées. Cependant «Phyo 636 », résistant, se révélant beaucoup plus inductible que «Yolo Wonder , sensible, nous allons étudier la réponse de 3 autres lignées résistantes.

\section{B. Réponse de plusieurs sources de résistance à diffé- rentes doses de la fraction $\mathbf{G} 4$ (fig. 1, tabl. 2)}

Les feuilles adultes mises en survie sur l'eau ont toutes été contaminées par un fragment calibré de mycélium. Dans le cas de variétés résistantes, cette façon de procéder s'avère indispensable si l'on veut observer des symptômes.

Quelle que soit la variété de piment, la concentration de $12 \mu \mathrm{g}$ de G4 par feuille assure la meilleure protection (fig. 1). Nous retrouvons un résultat déjà mentionné (tabl. 1).

Parmi les 5 variétés à l'essai, le matériel le plus inductible est la lignée « L 29 » (54 p. 100) puis, en ordre décroissant, « Phyo 636 » (59 p. 100), «PM $217 »(65$ p. 100), «CM 334 » (76 p. 100) et «Yolo Wonder » (79 p. 100). Mentionnons enfin qu'après dépôt de la fraction G4 et 
TABLEAU 1

Indice de sensibilité des organes foliaires de plantules de piment à $\mathrm{P}$. capsici après induction par la fraction G4 (100 p. 100 = organes foliaires totalement contaminés). Influence de la dose d'inoculum (souche 15) et de la quantité de G4 Sensibility index of pepper foliar organs to Phytophthora capsici after induction by G4 elicitor $(100$ p. $100=$ control entirely contamined $)$ Influence of inoculum quantity (isolate 15) and G4 quantity

Pour chaque variété et chaque organe foliaire (cotylédons ou feuilles), les résultats ont été interprétés statistiquement. Pour l'effet dose d'inoculum se reporter aux lettres de l'alphabet latin (lignes horizontales), pour l'effet dose d'éliciteur à celles de l'alphabet grec (colonnes verticales). Probabilité au seuil de 1 p. 100; Test de Duncan

Duncan test at the level 0,01 for the comparison of inoculum quantity (latin alphabet) and G4 quantity (greek alphabet)

\begin{tabular}{|c|c|c|c|c|c|c|c|c|c|c|}
\hline \multirow{3}{*}{$\begin{array}{r}\begin{array}{c}\text { par organe } \\
\text { foliaire }\end{array} \\
\begin{array}{r}\mathrm{Nb} \text { de } \\
\text { zoospores }\end{array}\end{array}$} & \multicolumn{6}{|c|}{ Yolo Wonder } & \multicolumn{4}{|c|}{ Phyo 636} \\
\hline & \multicolumn{3}{|c|}{ Cotylédons } & \multicolumn{3}{|c|}{ Jeunes feuilles } & \multicolumn{2}{|c|}{ Cotylédons } & \multicolumn{2}{|c|}{ Jeunes feuilles } \\
\hline & 800 & 5.000 & 10.000 & 800 & 5.000 & 10.000 & 5.000 & 10.000 & 5.000 & 10.000 \\
\hline 24 & ${ }_{32}^{\alpha}$ & $\alpha_{59}^{b}$ & ${ }_{64}^{\alpha}$ & ${ }_{21}^{\alpha}{ }^{a}$ & $\begin{array}{l}\alpha \quad a b \\
38\end{array}$ & ${ }_{43}{ }^{b}$ & $\alpha_{19} \mathrm{a}$ & ${ }_{25} \mathrm{a}$ & ${ }_{0}^{\alpha}{ }^{\mathbf{a}}$ & ${ }^{\alpha}{ }_{2}^{\mathrm{a}}$ \\
\hline 12 & ${ }_{28}^{\alpha}$ & ${ }_{51}^{\alpha} \mathbf{b}$ & $\alpha_{63}{ }^{b}$ & ${ }_{20} \mathrm{a}$ & ${ }_{35}{ }^{a}$ & ${ }_{40}{ }^{a}$ & ${ }_{17}{ }^{a}$ & ${ }_{20}^{\alpha} b$ & ${ }^{\alpha}{ }^{a}$ & $\begin{array}{ll}\alpha & \\
& \end{array}$ \\
\hline 6 & - & - & ${ }^{\alpha}{ }_{78}$ & $\beta_{56}{ }^{a}$ & ${ }^{\beta} 60^{a}$ & ${ }_{67}{ }^{\mathrm{a}}$ & - & ${ }_{39}$ & ${ }_{0}^{\alpha}{ }^{a}$ & ${ }^{\alpha \beta}{ }_{7}^{\mathrm{a}}$ \\
\hline 3 & ${ }^{\beta}{ }^{a}{ }^{a}$ & ${ }_{93}^{b}$ & ${ }_{100}^{b}$ & ${ }_{67}^{\beta \gamma} \mathrm{a}$ & $\gamma_{100}^{b}$ & $\gamma_{100}^{b}$ & $\beta_{40}{ }^{a}$ & $\beta_{85} \mathrm{~b}$ & ${ }^{\alpha} 2^{a}$ & ${ }^{\beta} 24$ \\
\hline $\begin{array}{l}0 \\
\text { (eau) }\end{array}$ & $\gamma_{100}$ & ${ }_{100}$ & ${ }_{100}$ & $\gamma_{84}$ & $\gamma_{100}$ & $\gamma_{100}$ & $\gamma_{81}$ & ${ }_{93}$ & ${ }^{\beta} 87$ & $\gamma_{100}$ \\
\hline
\end{tabular}

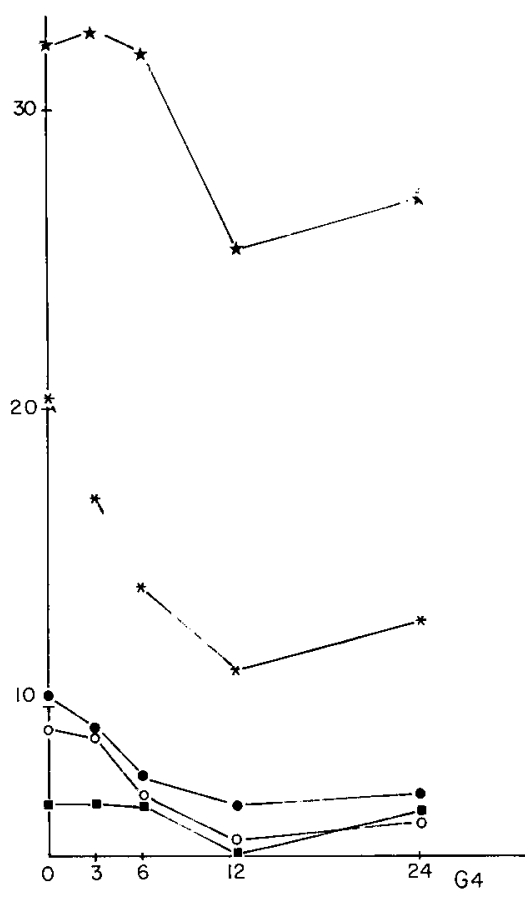

Figure 1

Diamètre moyen en $\mathrm{mm}$ de la zone attaquée sur feuilles adultes de 5 variétés de piment après induction par la fraction $G 4$ à 4 concentrations différentes ( $\mu \mathrm{g} /$ feuille); contamination par la souche 15

Average diameter ( $\mathrm{mm}$ ) of necrosis on leaves of five pepper lines after induction by different concentrations of $G 4$ elicitor ( $\mu \mathrm{g} / \mathrm{leaf}$ ).

$\star$ Yolo Wonder

* $L 29$

O Phyo 636

- PM 217

- CM 334
TABLEAU 2

Diamètre de la nécrose (en p. 100 du témoin sans éliciteur) sur feuilles adultes de 5 lignées de piment après induction par diverses concentrations de la fraction 64 (contamination par mycélium de la souche 15 sur blessure du limbe) Pour chaque variété, l'effet de la dose G4 est interprété selon le test de Duncan au seuil de probabilité de 1 p. 100

Necrosis diameter in p. 100 of corresponding control on leaves of 5 pepper lines after induction by several concentrations of G4 elicitor (mycelial contamination by isolate 15 of wounded leaves)

Duncan test at the level 0,01 for the comparison of G4 quantity

\begin{tabular}{lccccc}
\hline \hline & \multicolumn{4}{c}{ Quantités de G4 en $\mu$ g par feuille adulte } \\
\cline { 2 - 6 } & 0 & 3 & 6 & 12 & 24 \\
\hline $\begin{array}{l}\text { Yolo } \\
\text { Wonder }\end{array}$ & $100_{\mathrm{a}}$ & $101_{\mathrm{a}}$ & $99_{\mathrm{a}}$ & $79_{\mathrm{b}}$ & $84_{\mathrm{b}}$ \\
\hline Phyo 636 & $100_{\mathrm{a}}$ & $96_{\mathrm{a}}$ & $76_{\mathrm{b}}$ & $59_{\mathrm{c}}$ & $66_{\mathrm{b}}$ \\
\hline PM 217 & $100_{\mathrm{a}}$ & $88_{\mathrm{b}}$ & $73_{\mathrm{c}}$ & $65_{\mathrm{d}}$ & $68_{\mathrm{c}}$ \\
\hline L29 & $100_{\mathrm{a}}$ & $83_{\mathrm{b}}$ & $68_{\mathrm{c}}$ & $54_{\mathrm{d}}$ & $63_{\mathrm{c}}$ \\
\hline CM 334 & $100_{\mathrm{a}}$ & $100_{\mathrm{a}}$ & $98_{\mathrm{a}}$ & $76_{\mathrm{b}}$ & $97_{\mathrm{a}}$ \\
\hline \hline
\end{tabular}

en l'absence de contamination, les feuilles de «PM 217 » et « CM 334 » ont tendance à " nécroser ", tandis que " Yolo Wonder », "Phyo 636 » et « L 29 " ne manifestent aucun signe de phytotoxicité. Ce type de symptômes 
ne peut être confondu avec les dégâts provoqués par le champignon.

\section{Importance de la souche contaminatrice (tabl. 3).}

Dans le tableau 3, les 8 souches de $P$. capsici sont classées par ordre croissant d'agressivité sur feuilles adultes. Cet ordre, approximativement le même chez « Yolo Wonder " et « Phyo 636 » (cependant à un degré de sensibilité moindre dans ce dernier cas), présente des différences non négligeables avec le classement sur tiges qui, selon CLERJEAU et al. (1976), est le suivant : 75, 101, 13, 15, 107, 94, $91,197$.

Sur feuilles de "Yolo Wonder », la fraction G4 assure une diminution du diamètre des lésions de l'ordre de $25 \mathrm{p}$. 100 en moyenne, quelle que soit la souche de $P$. capsici. En revanche, chez « Phyo 636 », l'effet obtenu est non seulement plus important (environ 40 p. 100), mais encore plus dépendant des souches ; faible avec les souches 75, 94, 101 et 197, il est nettement plus important avec les souches 13 , 15,91 et 107.

Chez la variété résistante, l'appréciation de l'état induit apparaît fortement conditionnée, lors du test de contrôle, par le choix de la souche contaminante. Ce choix ne peut

\section{TABLEAU 3}

Indice de sensibilité en p. 100 des feuilles adultes de "Yolo Wonder 》 et « Phyo 636 " à plusieurs souches de P. capsici après induction par la fraction $G 4$ (12 $\mu \mathrm{g}$ /limbe

contamination par mycelium sur blessure de limbe

Pour chaque variété (colonnes verticales), le contrôle

par différentes souches de l'état induit a été interprété statistiquement au seuil de 1 p. 100 (test de Duncan)

Sensibility index on "Yolo Wonder " and "Phyo 636 " leaves to several Phytophtora capsici isolates after induction by G4 elicitor

(12 $\mu \mathrm{g} /$ leaf; mycelial contamination of wounded limb).

Duncan test at the level 0,01 for the comparison of Phytophtora capsici isolates

\begin{tabular}{llllll}
\hline $\begin{array}{l}\text { Lignées } \\
\text { Souches } \\
\text { de } \\
P . \text { capsici (1) }\end{array}$ & \multicolumn{2}{c}{$\begin{array}{c}\text { Yolo } \\
\text { Wonder }\end{array}$} & & Phyo 636 \\
\hline 15 & 80 & a & 43 & ab \\
\hline 94 & 67 & a & 68 & d \\
\hline 75 & 72 & a & 71 & d \\
\hline 13 & 73 & a & 40 & a \\
\hline 101 & 79 & a & 71 & d \\
\hline 197 & 77 & a & 77 & d \\
\hline 91 & 66 & a & 48 & ab \\
\hline 107 & 81 & a & 52 & bc \\
\hline Moyennes & 74,37 & a & 58,75 & c \\
\hline
\end{tabular}

(1) Classement par ordre croissant d'agressivité sur feuilles de "Yolo Wonder ": $15(6,2 \mathrm{~mm}), 94(7,3 \mathrm{~mm}), 75(7,5 \mathrm{~mm}), 13(8,6 \mathrm{~mm}), 101$ $(10,5 \mathrm{~mm}), 197(10,8 \mathrm{~mm}), 91(11 \mathrm{~mm}), 107(11,9 \mathrm{~mm})$.

Les chiffres entre parenthèses représentent les diamètres de zone attaquée. pas reposer sur le critère de l'agressivité, une souche fortement agressive sur feuille comme la 107 pouvant être aussi révélatrice de l'état induit qu'une faiblement agressive comme la 15. L'effet de protection observé peut néanmoins être atténué par la dose d'inoculum (voir plus haut tabl. 1).

\section{DISCUSSION - CONCLUSION}

Au cours de cette étude, nous avons apprécié la capacité d'induction de résistance des organes foliaires de piment par la fraction G4 issue du filtrat de culture de la souche 15-12-A de $P$. capsici.

Dans tous les cas, nous avons observé un effet de protection dont l'importance est fonction de plusieurs facteurs : quantité de G4, variétés de piment, dose d'inoculum et choix de la souche contaminatrice utilisée dans les contrôles biologiques.

En ce qui concerne la quantité de G4, la protection optimale est assurée par une concentration relativement faible, de l'ordre de 10-15 $\mu \mathrm{g}$ par site de dépôt de la goutte d'éliciteur ; si cette concentration diminue, l'effet est moins net ; si elle augmente, il en va de même avec, en plus, des risques de phytotoxicité. Ce phénomène " dose " a déjà été observé dans le cas de stimulation d'activité PAL de suspensions cellulaires par des «éliciteurs » fongiques (ALBERSHEIM \& VALENT, 1978 ; DIXON et al., 1981) et tout récemment dans le cas d'accumulation de glycoprotéines pariétales riches en hydroxyproline (ROBY, 1981).

Les feuilles des variétés de piment résistantes à $P$. capsici s'induisent mieux que celles des variétés sensibles comme "Yolo Wonder ». Citons le haut niveau de performance de « Phyo 636 » et surtout de la lignée « L 29 ». D'après ce que nous pouvons savoir par ailleurs (MOLOT et al., $1980 \mathrm{~b}$ ), la protection induite par éliciteur ne s'accompagne d'aucune synthèse de capsidiol. Il est curieux de constater qu'en infection classique sur tige, la lignée « L 29 » est celle qui en produit les plus faibles quantités (PoCHARD et al., 1983). Par ailleurs, des études récentes ont montré que cette lignée avait une remarquable aptitude à réduire la vitesse de progression du champignon dans la tige entre le $3^{\mathrm{c}}$ et le $7^{\mathrm{e}}$ jour suivant l'infection, à $22^{\circ} \mathrm{C}$ comme à $32^{\circ} \mathrm{C}$ (PoCHARD et al., 1983). Cette composante de la résistance appelée inductibilité (POCHARD \& DAUBEZE, 1980) semble donc pouvoir s'exprimer aussi dans le feuillage en réponse à des fractions élicitrices. S'il en est ainsi, on peut concevoir un programme de sélection basé sur la recherche de l'inductibilité maximum mesurée par des tests simplifiés conduits sur des feuilles en survie, traitées ou non par des fractions élicitrices.

Pour mcttre en évidence le phénomène d'induction par la fraction $\mathrm{G} 4$, il est important de réaliser la contamination artificielle avec de faibles quantités d'inoculum. Si celles-ci augmentent, l'effet de protection diminue et peut même s'annuler. La fraction G4 ne confère donc pas l'immunité absolue ; il y a simplement élévation du niveau général de résistance, ce niveau pouvant toujours être surmonté par des doses massives d'inoculum.

En contamination artificielle par du mycélium, nous avons montré, sur feuilles de la variété résistante "Phyo 636 ", le rôle de la souche contaminatrice. Mais, à l'opposé de ce qui se passe lorsqu'on augmente la dose d'inoculum, l'effet d'induction n'est pas nécessairement mieux révélé avec des souches peu agressives. Aucune corrélation ne semble exister entre l'agressivité d'une souche et son comportement sur tissus induits. 
Le travail effectué ici avec la fraction $\mathrm{G} 4$ de la souche 15-12-A mériterait d'être repris avec la fraction correspondante issue d'autres souches de $P$. capsici ou de champignons différents. Il serait intéressant de le généraliser à d'au- tres plantes que le piment. Ces études se poursuivent actuellement.

Reçu le 15 juillet 1982. Accepté le 29 novembre 1982.

\section{RÉFÉRENCES BIBLIOGRAPHIQUES}

\begin{abstract}
Albersheim P., Valent B., 1978. Host-pathogen interactions in plants. Plants, when exposed to oligosaccharides of fungal origin, defend themselves by accumulating antibiotics. J. Cell. Biol., 78, 627-643.

Clerjeau M., Pitrat M., Nourrisseau J.-G., 1976. La résistance du piment à Phytophthora capsici. IV. Etude de l'agressivité de divers isolats, au niveau des feuilles, des tiges et du collet de plantes sensibles et résistantes. Ann. Phytopathol., 8 (4), 411-423.

Coulomb Ph., Molot P.-M., Mas P., Conus M., Ferrière H., 1980. Mise en évidence d'une induction de capsidiol et de résistance chez Capsicum annuum par certaines fractions cellulaires obtenues après fractionnement du mycélium de Phytophthora capsici. C.R. Acad. Sci. Paris, t. 290, D, 275-277.

Dixon R.A., Dey P.M., Murphy D.L., Whitehead I.-M., 1981. Dose responses for Colletotrichum lindemuthianum elicitor-mediated enzyme induction in French bean cell suspension cultures. Planta, 151, 272-280.

Guererro-Moreno A., Laborde J.-A., 1980. Current status of pepper breeding for resistance to Phytophthora capsici in Mexico. Eucarpia Capsicum working group, $4^{e}$ congrès, 14-16 octobre 1980, Wageningen, p. 52-56.

Molot P.-M., Clerjeau M., Mas P., Ricci P., 1977. Rôle du capsidiol dans la résistance génétique du piment au Phytophthora capsici. Capsicum 77, $3^{e}$ congrès Eucarpia Piment, 5-8 juillet 1977, Montfavet,
\end{abstract} 137-146.
Molot P.-M., Mas P., Conus M., Ferrière H., 1980 a. La résistance du piment à Phytophthora capsici. VIII. Protection des organes foliaires après mise en survie sur un filtrat de culture du parasite. Caractérisation des conditions d'expression du phénomène. Ann. Phytopathol, 12 (2), 95-107.

Molot P.-M., Staron T., Mas P., 1980 b. La résistance du piment à Phytophthora capsici. VIII. Induction de résistance et.de capsidiol chez Capsicum annuum avec des fractions obtenues à partir de filtrats de culture et du mycélium de Phytophthora capsici. Ann. Phytopathol., 12 (4), 379-387.

Pochard E., Daubeze A.-M., 1980. Recherche et évaluation des composantes d'une résistance polygénique : la résistance du piment à Phytophthora capsici. Ann. Amélior. Plant., 30 (4), 377-398.

Pochard E., Molot P.-M., Dominguez G., 1983. Etude de 2 nouvelles sources de résistance à Phytophthora capsici Léon. chez le piment : confirmation de l'existence de 3 composantes distinctes dans la résistance. Agronomie, 3 (4), 333-342.

Roby D., 1981. Induction par des éliciteurs fongiques, via l'éthylène, de réactions associées à la défense des plantes. Thèse $3^{\mathrm{e}}$ cycle, Univ. Paul Sabatier, Toulouse, $67 \mathrm{p}$. 